\title{
COGNITIVE MODELING APPROACH FOR DEALING WITH CHALLENGES IN CYBER-PHYSICAL SYSTEMS
}

\author{
RÓBERT ADRIAN RILL ${ }^{(1,2)}$ AND ANDRÁS LŐRINCZ ${ }^{(1)}$
}

\begin{abstract}
In this paper, inspired by our previous works, we propose an architecture for the design and realization of cyber-physical systems (CPS) that considers the spatio-temporal context of events, promotes anomaly detection, facilitates efficient human-computer interaction and is capable of discovering novel human and/or machine knowledge. We view deep neural networks as smart sensors and sensory data from the environment represents the semantic and episodic input to a consistency seeking component of the cyber-space. Starting from a knowledge base infused with a deterministic world assumption, this module can detect anomalies and correct estimation errors by combining the outputs of multiple sensors. We also exploit an episodic description of ongoing situations by integrating temporal segmentation with kernel and low-dimensional embedding based methods. We demonstrate parts of the architecture through illustrative examples on our self-collected driving dataset. Our framework can be related to cognitive science foundations and may facilitate reliable functioning of CPS through integrating traditional AI and deep learning methods with deterministic models and reasoning tools. We expect that such knowledge base and cognition driven approaches of joining deep neural networks will be adopted in complex CPS. This looks like a scalable, and beneficial match between human knowledge and the exploding deep learning technologies.
\end{abstract}

\section{INTRODUCTION}

Cyber-physical systems (CPS) are complex structures of interacting physical and computational components, where the physical processes are controlled or monitored by computer-based algorithms. They are networks of sensors and robotic components equipped with advanced mechanisms and managed by intelligent software solutions, often including humans in the control loop.

Received by the editors: December 18, 2018.

2010 Mathematics Subject Classification. 68T01, 93A15.

1998 CR Categories and Descriptors. C.3 [Computer Systems Organization]: Special-purpose and Application-based Systems; I.2.0 [Computing Methodologies]: Artificial Intelligence - General.

Key words and phrases. cyber-physical system, human-computer interaction, anomaly detection, episodic description, consistency seeking. 
Requirements of a CPS include usability, functionality, robustness, efficiency, adaptability, safety and reliability. The design and realization of CPS call for the integration of theoretical models and engineering techniques originating from different disciplines. Examples and areas of application include autonomous driving systems, transportation, smart factories, intelligent manufacturing [12, 13], healthcare [25], civil infrastructure [3] (electrical power grid, water resources, communication systems, networked building control), assisted living (consumer appliances, intelligent homes), entertainment.

The digital revolution of the last decades has governed the long-term technological and economical trends of CPS. This approach integrates production in an intelligent computational space, leveraging the interconnectivity of selfadaptable machines, paving the way for the next generation manufacturing, namely Industry 4.0 [2], with a significant economic potential [13].

As multidisciplinary systems, CPS combine computation, communication and control technologies to conduct feedback control on widely distributed embedded computing systems [15]. They operate with inputs and feedback from/to the physical environment, which calls for workflow management with real-time performance requirements. Communication is realized in the form of sensor-actuator networks. The information represents the abstraction of the physical world and operations are events composed of states reported by sensors/humans and actions performed by actuators/humans. CPS realize the autonomous networking of embedded systems at large scales, and their interaction with environmental processes. This poses considerable challenges.

In this paper we build upon our previous works. In [16] we presented a framework of cognition that combines deep neural networks (DNNs) with facts and rules to correct recognition errors and obtain consistent and deterministic event descriptions. In our follow-up work [17], based on motivations from neuroscience and psychology, we introduced an architecture called declarative description that combines DNNs and unsupervised machine learning techniques to provide explanation and reasoning about ongoing situations in a spatio-temporal context. The contribution of the present paper consists in combining the concepts of our former efforts to propose a general architecture for the design and implementation of CPS. Our framework tackles the challenges of CPS: anomaly detection, efficient human-computer interaction and deterministic decision making and description of events. Furthermore, we view DNNs as smart sensors and illustrate parts of the architecture on our self-collected driving data as a simple CPS scenario.

The paper is organized as follows. Section 2 provides a background by reviewing challenges that CPS face. Section 3 presents the two components that extend the traditional sensor-controller-actuator CPS network, namely 
consistency seeking and episodic description. Section 4 describes our illustrative examples, followed by a discussion in Section 5. Finally, Section 6 concludes the paper.

\section{Background: CPS Challenges}

2.1. Anomalies and decision making. CPS aim for a high level of certainty in a narrow context, i.e. they are goal-oriented, designed with a well defined purpose, and they tightly integrate physical and cyber system aspects both at design time and during operation [3]. However, CPS are exposed to unexpected events, i.e. anomalies, which need to be recognized and dealt with in real time and with extreme care in order to limit false alarms and unobserved faults.

Real-time responsiveness of CPS to the environment is also related to timing behavior, which emerges from the combination of software and hardware platforms [11]. Predictable and reliable real-time performance is difficult to achieve because of the lack of temporal semantics and adequate concurrency models in computing [1]. Lee [10] summarized this thought as "the program does not express aspects of behavior that are essential to the system". CPS must have unified time, trust quantification and communication mechanisms at the system level [15].

2.2. Deterministic models: knowledge-base. In order to realize CPS, we need smart analytical tools to transform experience-based knowledge into evidence-based decision making for sustainable and reliable operation [25]. Components of a CPS come from multiple vendors in diverse engineering disciplines with distinct domain expertise [11, 15]. A holistic approach is required that integrates the physical and computational infrastructures into one unified model [14] for supporting real-time, reliable and autonomous decision making.

It is the power of deterministic models that gave scientists the ability to design control systems [11]. In CPS simulation environments they facilitate control algorithm development and testing, before the deployment into hardware [22]. Starting from a knowledge base that includes domain expertise, constraints, reasoning tools, facts and rules of the physical world, the realization of predictable and understandable models is possible. Moreover, a comprehensive knowledge about its own dynamic structure and the infrastructure of the whole system results in self-monitoring and self-aware CPS [2].

A knowledge-based decision making component can deal with the dynamic behavior: (i) it can perceive and control the environment, analyze observations due to the intelligent data management capabilities of the cyber space, and (ii) can communicate in efficient ways with other modules through wireless sensor networks [3] and with humans through intelligent user interfaces [13]. 
2.3. Deep learning in CPS. Novel engineering solutions exploiting AI and deep learning are improving at a remarkable pace. AI-based technologies are being developed for smart machine control [12]. In a networked CPS setting with interactions with the physical environment and humans, conditions are dynamically changing. This requires greater flexibility in modeling and optimized decision making. The advancements of the past years creates an opportunity to add data driven intelligence to the CPS processes.

To complement the available knowledge base, one can make use of DNNs. In CPS the measurement and monitoring of physical processes (temperature, pressure or mechanical movements, for instance) are done by sensors which convert physical or electrochemical properties into an electrical output signal [2]. So-called smart sensors are utilized for monitoring and control mechanisms of the environment. They enable reliable, accurate and automated data collection with minimal maintenance efforts and flexible networking. In this sense typical DNNs are sensors that monitor characteristics of CPS components and the environment, and provide a signal as output, which serves decision making and control. In the rest of the paper, smart sensors and DNNs will be referred to collectively as feedforward input-output (FIO) systems.

To summarize, DNNs may extend the set of intelligent sensors for increasing flexibility and adaptability in CPS. However, it is still required that humans remain in the loop in order to complement autonomy technologies for maximizing performance and limiting risks [21].

2.4. Humans-in-the-loop. CPS often involve humans in decision making and control loops. Either (i) they have supervisory roles and directly control the system, or (ii) the system passively monitors humans, collects data to be analyzed and takes actions if necessary. There are also different levels of human control depending on how large the task load is for decision making and how active the involvement is in the autonomy of making decisions [21].

In either case, several challenges need to be tackled. The modeling of human behaviour is a difficult task due to the complex physiological, psychological and behavioral aspect of humans [19, 20]. Furthermore, the probability of human error causing a system failure can be high due to a variety of reasons [21]. Therefore, robust CPS systems call for real-time predictive models that are able to recognize dangerous situations, control the outcomes, maintain stability and accuracy and adapt to changing human behavior.

Human behavior models need to be incorporated into the system architecture itself and, as several researchers suggest, human interaction will have a critical role in the foreseeable future (see, e.g., [21]). 


\section{Methods: Cognitive ARChitecture for CPS}

3.1. Events in deterministic environments. CPS deal with scheduled operations and involve decision making in a goal-oriented context, often with humans in the loop. Any operation in the physical system can be described as an initial state and an action to be executed leading to a final desired state, where the action may involve several sub-processes (see Figure 1). We call the transition from the actual state to another one after executing the action an event, or episode. Two remarks have to be made. Firstly, the final state may differ from the desired state giving rise to an error term. We will turn back to this point later in the paper. Secondly, episodes can have different properties depending on their interrelationships: they can follow each other, can be concurrent, can be composed of other smaller episodes, can be combined into higher-order episodes.

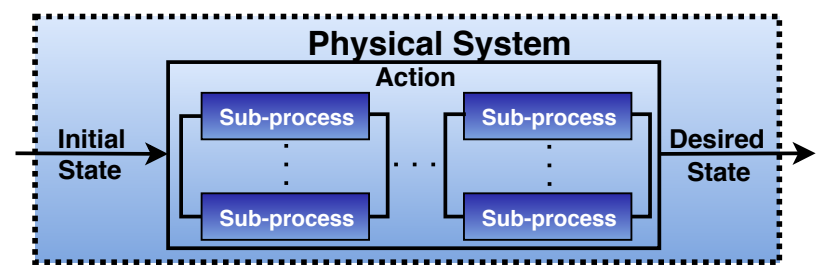

FiguRE 1. Operations in a physical system as events.

An event is independent if the state-action-state transition is not affected by other concurrent actions. An event is non-stochastic if the desired state is reached with $100 \%$ probability in the prescribed execution time. Deterministic behavior presumes independent and non-stochastic events. However, because of their complexity and interactions with the environment, events are less likely to be non-stochastic. Stochastic problems arise from anomalies, unpredictability of execution time and uncertainties from the environment, e.g. non-modelled side-effects of the surroundings.

Problem solving in such complex systems is a combinatorial problem and lowering of the number of variables is highly desired due to the exponential dependence of the state space on those. Because CPS are goal oriented, the deterministic world assumption points towards the ability of reaching goals and desired states with $100 \%$. If anomalous events/episodes occur, deterministic models built upon the available knowledge base provide real-time decision making capabilities for recognizing and resolving them. Moreover, the spatiotemporal dependencies of the processes in a complex CPS constrain possible state-desired state pairs limiting the number of variables to be considered. 
3.2. Consistency seeking component of the cyber space. In a typical CPS setting, sensors collect information from the environment, the cyber layer stores information and carries out abstract computations to examine the collected signals, controllers make decisions, which are transmitted to the actuators in order to change the physical processes $[3,14]$.

To extend the cyber space capabilities, we propose a consistency seeking module for decision making and control in a spatio-temporal context, making use of the available knowledge base. The contextual environment is given by sensory information - events taking place at a given location at a given time - and also incorporates the knowledge of experts. The sensory input is converted by the FIO systems into semantic and episodic input and this enters the consistency seeking module of the cyber space (see Figure 2). The semantic and episodic output of the consistency seeking component may be used to overwrite the collected signal to obtain consistent representations of the physical processes, or may be combined to produce the actual state. The difference between the actual and desired state gives rise to an error term, used to induce changes in the physical world.

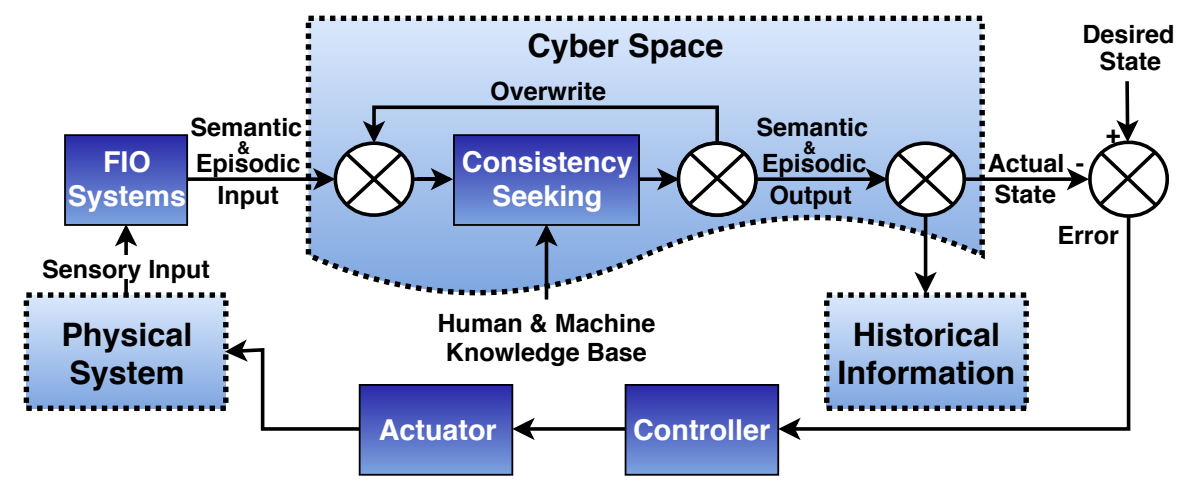

Figure 2. The traditional closed-loop sensor-controller-actuator CPS network extended with a consistency seeking component.

The consistency seeking component can facilitate anomaly detection. We propose to combine the outputs of multiple FIO systems to discover and resolve conflicts, to take into account the spatio-temporal context and the available knowledge base for improving recognition. Facts and rules, the structure and meaning of information, semantic relations among the components of the physical and cyber infrastructures can be injected into ontologies that also describe the interdependencies across the cyber-physical boundary [14].

The consistency seeking component of our architecture assumes a deterministic world. In stochastic environments other approaches might be necessary, 
e.g. probability maximization. However, if sufficient information is available, the deterministic world assumption holds, and prediction errors and persisting contradictions should lead to searches for finding the missing causes.

To summarize, consistency seeking and the assumption of determinism together become powerful tools for anomaly detection and learning, if we can recognize components and have episodic knowledge about their spatio-temporal relationships.

3.3. Episodic description of ongoing situations. Anomalies are changes in behavior that negatively affect performance. They are outliers that correspond to short time intervals within a larger episode, e.g. texting while driving.

CPS require real-time capabilities to keep updated about the current states of physical devices and to intervene if necessarry in applications such as observation, monitoring, control, forecasting [15]. Therefore, in a CPS setting, sensors continuously monitor physical processes such as traffic information in intelligent transportation, patients' blood pressure or blood sugar level in the healthcare domain, soil temperature and humidity in environmental detection. In the physical world the passing of time is inevitable and concurrency of processes is naturally present. Our framework integrates these properties in the computing capabilities of the cyber space.

Particularly, we propose to further extend the cyber space capabilities with an episodic description of ongoing situations illustrated on Figure 3. The sensory data and semantic and episodic output of the consistency seeking module are considered time series and temporal segmentation is applied. The result is a series of episodes, which can be consecutive or overlapping. Segmentation is followed by the comparison of episodes with each other, and the obtained similarity vectors are embedded into low-dimension. The resulting clusters can be further inspected by intelligent algorithms or humans using smart interfaces.

Episodic description could be applied for example in manufacturing, where most production planning decisions are based on historical data [2]. If past information of a production machine is provided in terms of time series, the episodic description module is capable to track the changes and infer additional knowledge by searching for similarities with other machine records and analyzing performance. This gives the possibility for the cyber space to predict future behavior. Another example is in the healthcare domain, where semantic knowledge needs to be integrated with manually and automatically collected low-cost clinical patient data towards clinical decision support [20, 25]. Temporal segmentation and/or clustering of episodes can be applied to historical information of patient data to analyze behavior patterns and predict possible future diseases or recovery status. 


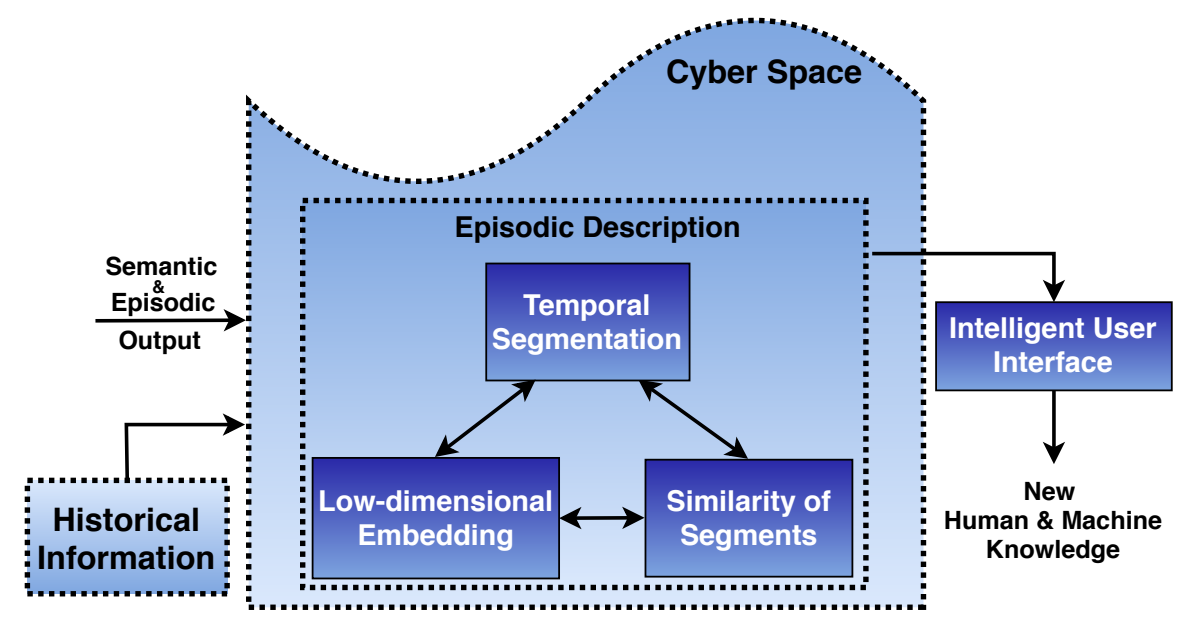

Figure 3. Extension of the cyber space with an episodic description component to detect anomalies and to bring temporality into focus.

\section{Results: DESCRIPTION OF ILLUSTRATIVE EXAMPLES}

Autonomous driving represents one area of application for CPS [5, 7]. Our choice of the CPS aims the illustration of correcting sensory observations via consistency seeking. Our purpose was well served by means of self-collected data. Reasons include risk considerations, the lack of data about monitored humans, and the simplicity of illustration during driving, as a CPS scenario. We recorded videos while driving between Budapest and Martonvásár in Hungary, using a spherical camera attached to the dashboard of the car ${ }^{1}$.

To illustrate the consistency seeking module of our architecture we extracted frontal view videos from our collected spherical videos and ran state-of-the-art DNNs on them. Figure 4 shows how the consistency seeking module can combine the outputs of two state-of-the-art DNNs to correct estimation errors and obtain new samples for fine-tuning: (i) the OpenPose version of the Convolutional Pose Machine (CPM) [24] is used to estimate body joint coordinates and (ii) the mismatches are corrected by predicting the movement of pixels from one frame to the next with optical flow, using the FlowNet2 version [9].

Figure 5 illustrates how the consistency seeking module can combine the outputs of DNNs to complement each other: (i) the state-of-the-art Yolo [23] object detector is used to recognize vehicles on videos; (ii) if the detection is lost on one frame, FlowNet2 [9] can be applied to predict the movement of the bounding box using the detection from the previous frame.

\footnotetext{
${ }^{1}$ Ricoh R Development Kit: https://ricohr.ricoh/en/
} 

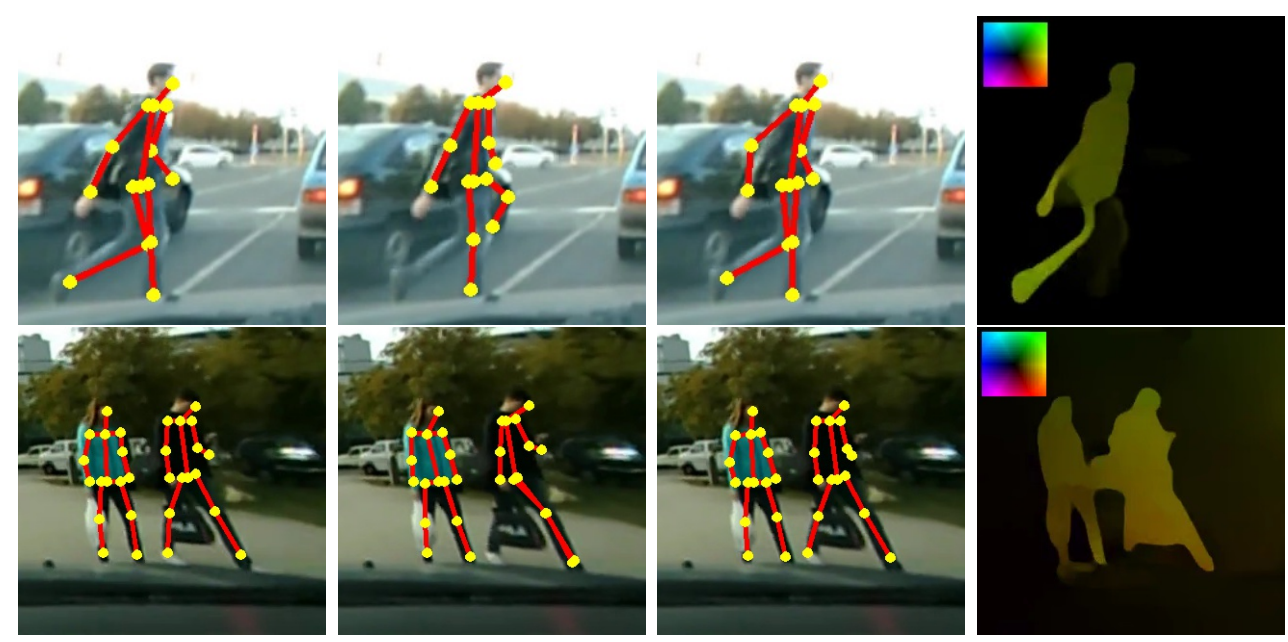

Figure 4. Correction of Convolutional Pose Machine (CPM) results by optical flow (OF). Two instances are shown in rows. The columns from left to right are: current frame with CPM visualized, next frame with mismatched CPM, same frame as second column with corrected CPM, and $\mathrm{OF}$ from current to next frame visualized. The square represents color coding of OF.

To demonstrate the episodic description component of our architecture we show how it can be applied to detect anomalies. Namely, we used it to recognize and cluster overtaking events, i.e. time segments when other cars pass by ours during driving. The spherical videos were pre-processed as follows: (i) extract side-views so that the car's side window is in the center of the frames, (ii) apply video stabilization to reduce the oscillation due to the movement of the camera, (iii) extract center of frames containing only the side window of the car, (iv) run FlowNet2 [9] on these small resolution videos and (v) compute OF features to obtain time-series subject to temporal segmentation. The features are 5-dimensional: for each frame we computed the two-bin histogram of the horizontal $\mathrm{OF}$ and choose the two bin heights and the three bin endpoints as features.

After the pre-processing procedure the steps of the episodic description were applied. Similarly to our previous works $[16,17,18]$, for temporal segmentation we used the Group Fused LASSO [4] method to detect change-points cooccurring across the dimensions of multivariate signals. Then segments were compared with the Global Alignment Kernel (GAK) technique [6], able to compare different length segments by using time warping. Finally, the t-SNE algorithm [26] was applied to embed the columns of the similarity matrix into low-dimensions. Figure 6 shows an example of temporal segmentation on a 

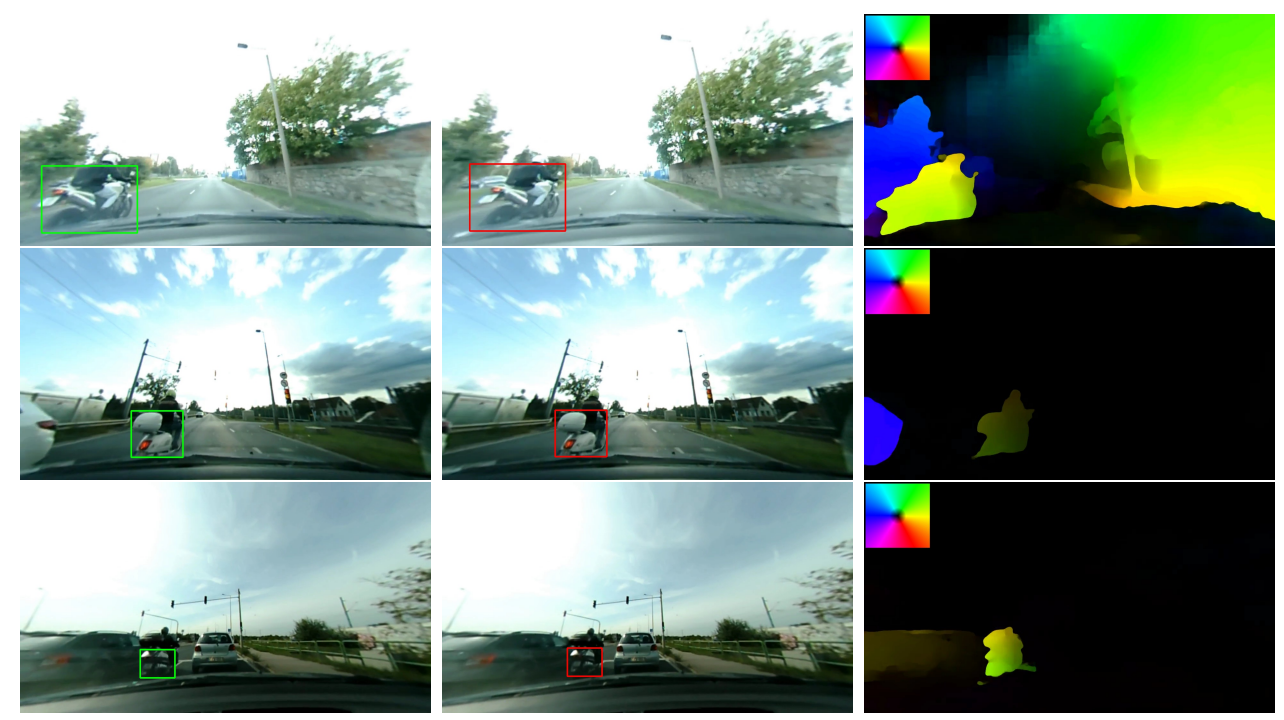

Figure 5. Complementing Yolo object detection by optical flow (OF). Three instances are shown in rows. The columns from left to right are: current frame with detected Yolo bounding box, next frame with predicted bounding box, OF from current to next frame visualized. The square represents color coding of $\mathrm{OF}$.

short video, where three overtaking episodes (around frames 310, 790, 960) are clearly detected.

For the purposes of illustrating the results of the whole episodic description pipeline, we selected a 30 minute long driving video and hand-annotated the overtaking and the stopped segments (this latter refers to the time intervals when our car was stopped in traffic). The results are displayed on Figure 7, where each point corresponds to a time segment, as determined by temporal segmentation. There are a total of 44 overtaking episodes in this video. They are clustered together and separated from the rest to a large extent (red points).

It must be noted that long episodes can be segmented into multiple parts by the GFLASSO algorithm. Therefore, trajectories are also visualized on Figure 7, i.e. temporally consecutive points are connected with lines to reveal the difference between the three categories from the small cluster. Continuous colored lines connect consecutive segments of the same overtaking episode and they settle in the upper small cluster, with only one exception: in the lower right part of the figure the lightblue trajectory - this actually corresponds to two separate very long and consecutive overtaking events. All points from the small cluster corresponding to stopped segments (black) have their next 


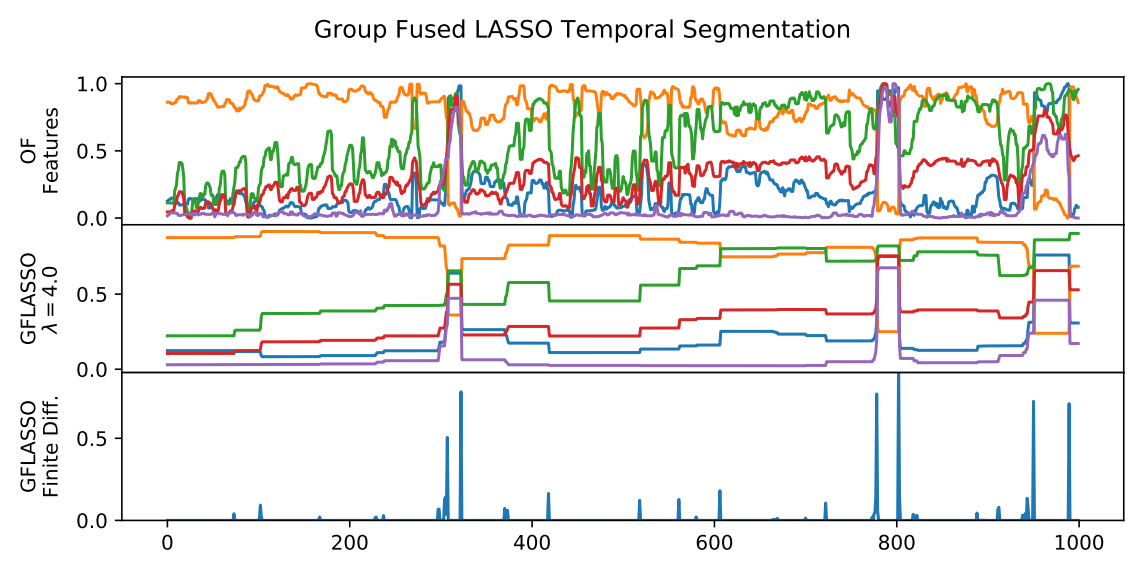

Figure 6. Example of Group Fused LASSO temporal segmentation on a 1000 frame long video. From top to bottom: raw Optical Flow (OF) features normalized, GFLASSO segmentation, finite differences of GFLASSO corresponding to change-points.

segment as stopped, connected by dashed grey lines (only three are shown for visual clarity). Dotted grey lines connect three other category points (blue) from the small cluster with their next segment: one is red inside and two are black outside the small cluster. Also three blue points from the small cluster have their next segment not overtaking nor stopped (these trajectories are not shown).

To sum up, assuming that stopped episodes are known and making use of trajectories, episodic description recognized the overtaking events with high probability as illustrated by Figure 7:

- 42 episodes out of 44 were separated from the rest in the small cluster (some of these episodes are composed from multiple segments but the trajectories settle in the small cluster);

- there are two false negatives (two long consecutive overtaking events connected by the lightblue line);

- there are three false positives (in the small cluster three blue points can not be excluded because their next segment is also blue).

Omitting the stopped segments from calculation and connecting the segments of the same event with trajectories, the problem of recognizing overtaking events can be treated as binary classification. Our relatively small example analyzed in detail happens to bring high accuracy (99\%), precision (93\%), recall (95\%) and F1 score (94\%) for classifying overtaking upon segmentation. 
t-SNE embedding

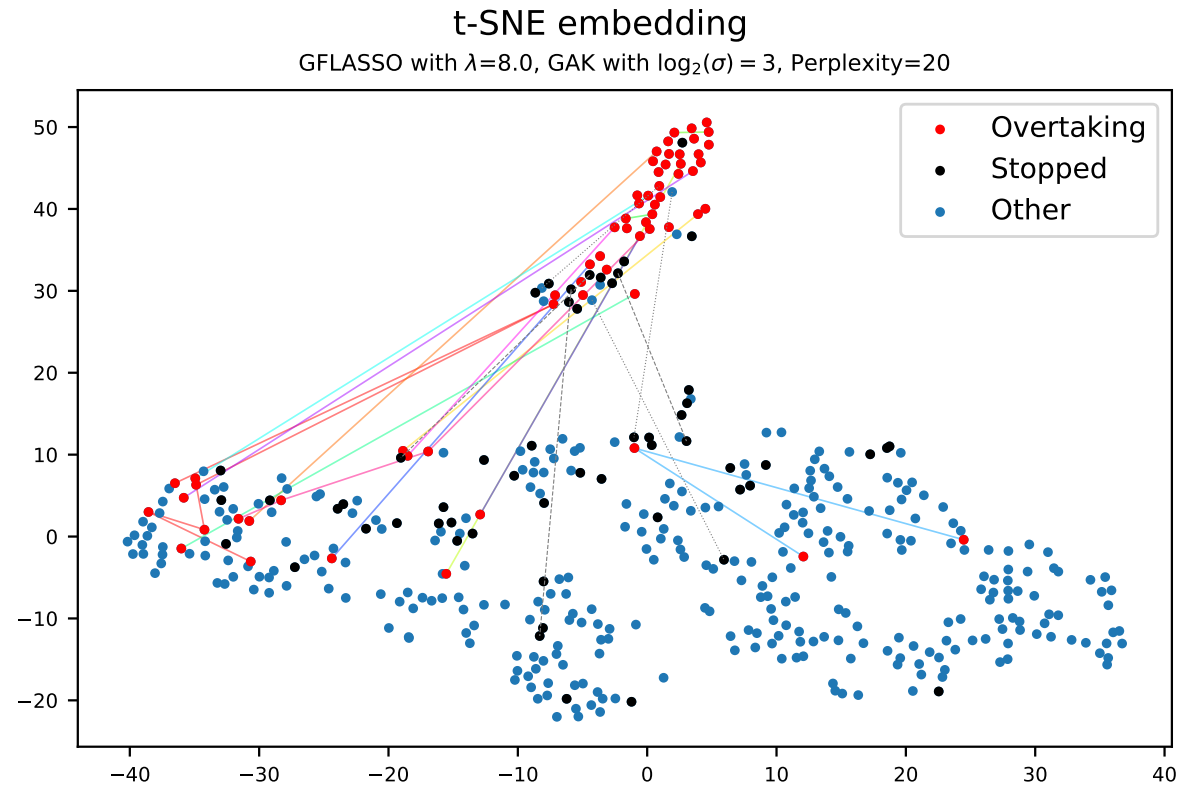

FiguRE 7. t-SNE embedding results for episodic description in the case of a 30 minute long video.

\section{Discussion}

Our illustrative examples show how the consistency seeking module can combine the outputs of multiple FIO systems to correct estimation errors and to complement each other, and how the episodic description component can be used for event/anomaly detection. The overtaking episode represents a dangerous situation in an autonomous driving system and should be recognized in time in order to take appropriate actions.

The episodic description involves events that concern observations and interactions between components. The events are localized in space and time and reflect the physical reality. This enables reasoning at higher levels while integrating with a human/machine knowledge base. Not only does episodic description bring temporality into focus, but it has another important consequence. Although large volumes of data are collected in a CPS, not all of it is relevant, thus reducing the region of interest is essential [3].

The architecture presented in this paper tackles the problem of combinatorial explosion by limiting the number of variables to be considered during decision making in two ways: (i) the consistency seeking component considers 
the spatio-temporal context, which constrains the number of possible statedesired state pairs, and (ii) the episodic description contributes to eliminating irrelevant intervals of the state space.

To give a simple example, the spatially limited OF between a few frames is an instance of the extremes of short episodes. It limits efficiently the space of potential future outcomes due to Newtonian laws. In case of object tracking it means that a bounding box may not appear at any given position in the next frames. Thus the set of potential positions of the bounding box may be reduced considerably due to the "OF episode". More is expected for deterministic processes in the long run if the potential next episodes are learned and can be identified during progress.

High accuracy, recall and F1 score values for event recognition mean that high probability warning signals can be provided to the driver. Furthermore, the driver may train the system for personal use if their behavior is monitored. See for instance the examples provided in [16], which include talking to a person in the car or over the phone, or drinking. In this case the driver can train the system conditioned on their behavior, by providing feedback if the warning signal is not needed. Moreover, if the attention of the driver is overloaded then the warning signal may be desired in the same or similar situations. Feedback should help the step-by-step learning about the proper level of interaction. This can be the subject of future data collection, since the spherical camera enables the monitoring of the driver as well.

We close this section by noting that present CPS researches are still at the beginning, and are mostly limited to specific applications. Further investigations and studies are required to realize a unified framework of computational and physical resources $[1,15]$. Since future CPS will cover aspects of social and economic lives [3], it is crucial to establish easy to abstract models so that the complexity of design can be reduced.

5.1. Relation to cognitive psychology. Our architecture can be related to fundamentals of the human cognitive system, namely declarative (semantic and episodic) and procedural memory (for details on this topic see, e.g., [8]). Semantic memory assumes a collection of facts and concepts encoded with specific meaning independently from the spatio-temporal context. Episodic memory on the other hand represents experiences and events in a serial form from which the situation can be reconstructed only if the surrounding context is also present. The procedural memory involves slow and gradual acquisition of skills that often occurs without conscious attention to learning. The concept of cognition is more than a single input-output mapping, it includes information processing, acquiring knowledge and the capability of reasoning. 
In relation to our architecture, every event is an episode and it can be saved in the episodic 'memory' for data mining, anomaly detection, model construction, and for learning to predict and control the event. This event may be concurrent with other events and it is probably embedded into a larger one. The method of dealing with an ongoing event is the procedure, composed of actions and sub-events. This procedure may evolve over time as knowledge is collected. Reliable functioning is possible if the semantic knowledge base is large enough. If not, than new concepts, sensors and additional control tools can be introduced to overcome disturbances of the events provided that the details of the event are comprehensible, time is available and the related costs and savings justify the effort.

\section{Conclusion}

In this paper, inspired by our previous works, we presented an architecture for CPS that can be related to fundamentals of the human cognition system. To extend the traditional sensor-controller-actuator network, we proposed two novel components to enhance the cyber space capabilities: (i) consistency seeking and (ii) episodic description. The consistency seeking module is capable of detecting anomalies and correcting estimation errors by combining the outputs of multiple smart sensors and/or deep neural networks taking into consideration the spatio-temporal context and the available knowledge base (facts, rules of the physical world, domain-specific ontologies etc.). The episodic description module considers timing behavior, combines sparsity, kernel and low-dimensional embedding methods for anomaly detection, facilitates efficient human-computer interaction and is capable of discovering novel human and/or machine knowledge. By integrating deep learning and traditional AI methods with reasoning tools and deterministic assumption, our approach facilitates understandable and reliable decision making.

Our framework points to goal-oriented systems, an essential property of CPS. It considers anomaly detection and their resolution, i.e. noticing discrepancies in space and time and seeking for consistency based on the assumption of determinism. We expect that such knowledge base and cognition driven approach of joining deep neural networks will be adopted in complex CPS.

\section{ACKNOWLEDGEMENT}

The research has been supported by the European Union, co-financed by the European Social Fund (EFOP-3.6.2-16-2017-00013, Thematic Fundamental Research Collaborations Grounding Innovation in Informatics and Infocommunications). AL was partially supported by the European Union, co-financed by the European Social Fund (EFOP-3.6.3-VEKOP-16-2017-00001). 


\section{REFERENCES}

[1] M. Atif, S. Latif, R. Ahmad, A. K. Kiani, J. Qadir, A. Baig, H. Ishibuchi, and W. Abbas. Soft computing techniques for dependable cyber-physical systems. CoRR, abs/1801.10472, 2018.

[2] C. Berger, A. Hees, S. Braunreuther, and G. Reinhart. Characterization of cyberphysical sensor systems. Procedia CIRP, 41:638-643, 2016. Research and Innovation in Manufacturing: Key Enabling Technologies for the Factories of the Future - Proceedings of the 48th CIRP Conference on Manufacturing Systems.

[3] M. Z. A. Bhuiyan, J. Wu, G. Wang, J. Cao, W. Jiang, and M. Atiquzzaman. Towards cyber-physical systems design for structural health monitoring: Hurdles and opportunities. ACM Trans. Cyber-Phys. Syst., 1(4):19:1-19:26, 2017.

[4] K. Bleakley and J.-P. Vert. The group fused Lasso for multiple change-point detection. working paper or preprint, 2011.

[5] A. Chattopadhyay and K.-Y. Lam. Security of autonomous vehicle as a cyber-physical system. In 2017 7th International Symposium on Embedded Computing and System Design (ISED), pages 1-6, 2017.

[6] M. Cuturi. Fast global alignment kernels. In Proceedings of the 28th International Conference on International Conference on Machine Learning, ICML'11, pages 929-936, USA, 2011. Omnipress.

[7] T. Dreossi, A. Donzé, and S. A. Seshia. Compositional falsification of cyber-physical systems with machine learning components. CoRR, abs/1703.00978, 2017.

[8] M. W. Eysenck. Fundamentals of Cognition. Psychology Press, 2012.

[9] E. Ilg, N. Mayer, T. Saikia, M. Keuper, A. Dosovitskiy, and T. Brox. Flownet 2.0: Evolution of optical flow estimation with deep networks. In 2017 IEEE Conference on Computer Vision and Pattern Recognition (CVPR), pages 1647-1655, 2017.

[10] E. A. Lee. Cyber physical systems: Design challenges. In 2008 11th IEEE International Symposium on Object and Component-Oriented Real-Time Distributed Computing (ISORC), pages 363-369, 2008.

[11] E. A. Lee. Cyber-physical systems: A rehash or a new intellectual challenge? Invited Talk in the Distinguished Speaker Series, sponsored by the IEEE Council on Electronic Design Automation (CEDA) held at the Design Automation Conference (DAC), Austin, Texas., 2013.

[12] J. Lee, B. Bagheri, and C. Jin. Introduction to cyber manufacturing. Manufacturing Letters, 8:11-15, 2016.

[13] J. Lee, B. Bagheri, and H.-A. Kao. A cyber-physical systems architecture for industry 4.0-based manufacturing systems. Manufacturing Letters, 3:18-23, 2015.

[14] J. Lin, S. Sedigh, and A. Miller. A Semantic Agent Framework for Cyber-Physical Systems, pages 189-213. Springer Berlin Heidelberg, Berlin, Heidelberg, 2011.

[15] Y. Liu, Y. Peng, B. Wang, S. Yao, and Z. Liu. Review on cyber-physical systems. IEEE/CAA Journal of Automatica Sinica, 4(1):27-40, 2017.

[16] A. Lőrincz, M. Csákvári, Áron. Fóthi, Z. Á. Milacski, A. Sárkány, and Z. Tősér. Towards reasoning based representations: Deep consistence seeking machine. Cognitive Systems Research, 47:92-108, 2018.

[17] Z. Á. Milacski, K. B. Faragó, A. Fóthi, V. Varga, and A. Lőrincz. Declarative description: The meeting point of artificial intelligence, deep neural networks, and human intelligence. In IJCAI/ECAI 2018 Workshop on Explainable Artificial Intelligence (XAI), 2018 . 
[18] Z. Á. Milacski, M. Ludersdorfer, A. Lörincz, and P. Van Der Smagt. Robust detection of anomalies via sparse methods. In Lecture Notes in Computer Science, volume 9491, pages 419-426. Springer Verlag, 2015.

[19] S. Munir, J. A. Stankovic, C.-J. M. Liang, and S. Lin. Cyber physical system challenges for human-in-the-loop control. In Presented as part of the 8th International Workshop on Feedback Computing, San Jose, CA, 2013. USENIX.

[20] W. Nilsen, E. Ertin, E. B. Hekler, S. Kumar, I. Lee, R. Mangharam, M. Pavel, J. M. Rehg, W. Riley, D. E. Rivera, and D. Spruijt-Metz. Modeling Opportunities in mHealth Cyber-Physical Systems, pages 443-453. Springer International Publishing, Cham, 2017.

[21] W. D. Nothwang, M. J. McCourt, R. M. Robinson, S. A. Burden, and J. W. Curtis. The human should be part of the control loop? In 2016 Resilience Week (RWS), pages 214-220, 2016.

[22] N. Pedersen, T. Bojsen, and J. Madsen. Co-simulation of cyber physical systems with hmi for human in the loop investigations. In Proceedings of the Symposium on Theory of Modeling $\mathcal{G}$ Simulation, TMS/DEVS '17, pages 1:1-1:12, San Diego, CA, USA, 2017. Society for Computer Simulation International.

[23] J. Redmon and A. Farhadi. Yolo9000: Better, faster, stronger. In 2017 IEEE Conference on Computer Vision and Pattern Recognition (CVPR), pages 6517-6525, 2017.

[24] T. Simon, H. Joo, I. Matthews, and Y. Sheikh. Hand keypoint detection in single images using multiview bootstrapping. In 2017 IEEE Conference on Computer Vision and Pattern Recognition (CVPR), pages 4645-4653, 2017.

[25] D. Sonntag, S. Zillner, S. Chakraborty, A. Lörincz, E. Strommer, and L. Serafini. The medical cyber-physical systems activity at EIT: A look under the hood. In 2014 IEEE 27th International Symposium on Computer-Based Medical Systems, pages 351-356, 2014 .

[26] L. van der Maaten and G. Hinton. Visualizing data using t-SNE. Journal of Machine Learning Research, 9:2579-2605, 2008.

(1) Faculty of Informatics, Eötvös Loránd University. H-1117 Budapest, Pázmány P. stny 1/C, Hungary. 3in Research Group, Martonvásár, Hungary.

(2) Faculty of Mathematics and Computer Science, BabeŞ-Bolyai University. 1 Mihail Kogălniceanu, RO-400084 Cluj-Napoca, Romania.

Email address: rillrobert@cs.ubbcluj.ro (ORCID iD: http://orcid.org/0000-00023004-7294), lorincz@inf.elte.hu (ORCID iD: https://orcid.org/0000-0002-1280-3447) 